\title{
DOES FLSE PERCEIVE ORGANIZATIONAL IMO IMPACT ON THEIR CUSTOMER ORIENTED BEHAVIOR?
}

\author{
Ahmed Ferdous, Deakin University, Australia \\ Michael Polonsky, Deakin University, Australia
}

\begin{abstract}
Frontline Service employees (FLSEs) are responsible for the effective delivery of services and thus are critical in determining how customers evaluate organizations. Given their focal role in the exchange process it is essential that marketing strategies and programs targeting FLSEs, ensure they (the FLSEs) deliver on organization's marketing programs and objectives. To achieve employee engagement with strategic actions, organizations develop internal marketing orientation (IMO) as a broad strategic approach which is designed to systematically understand and respond to employees' needs by delivering the right 'job' product. This is analogous to traditional market orientation (MO), where firms gather external information and then respond to external customers' needs. From the perspective of Internal Marketing (IM), it has been argued that even though management may craft the most carefully developed organizational internal market orientation (IMO), the successful implementation of these programs are contingent on how FLSE's respond to IMO as a general concept. It is therefore vital for management to develop IMO at the organizational level (i.e. organizational IMO) that is viewed positively by employees. However, to date FLSEs' perceptions of organizational IMO have been under-researched, which is somewhat surprising, even though some studies exist, given that it is FLSEs who determine whether IMO implementation succeed or fail. To address this gap, this paper develops and tests a conceptual model investigating if FLSE perception of organizational IMO impact on their job satisfaction (JS) and organizational identification (OI) and whether these in turn impacts on their customer oriented behaviors (COB) which is the ultimate focus of implementing IMO targeting FLSEs.
\end{abstract}

The hypotheses were tested using a sample of 295 frontline salespeople working for a large Bangladeshi general insurance company, using the bootstrapping bias corrected 95\% confidence interval procedure in SEM using AMOS 20 . The paper also uses the phantom-model approach to calculate specific indirect (i.e., mediated) effects of perception of organizational IMO on COB via JS and OI. The findings of this paper suggest that FLSE's perception of organizational IMO impact on their COB via JS and OI. This would mean that firms should foster both FLSE's OI as well JS as both are equally important for positively mediating the impact of perception of organizational IMO on FLSE's COB i.e. achieve broader organizational actions designed to improve customer outcomes.

References available upon request 